\title{
RESULTADOS DO TRATAMENTO DE FRATURAS CRÔNICAS PELA VIA ANTERIOR EM PACIENTES COM OSTEOPOROSE
}

\author{
RESULTS OF TREATMENT OF CHRONIC FRACTURES BY ANTERIOR APPROACH IN PATIENTS \\ WITH OSTEOPOROSIS
}

\section{RESULTADOS DEL TRATAMIENTO DE FRACTURAS CRÓNICAS POR LA VIA ANTERIOR EN PACIENTES CON OSTEOPOROSIS}

Fabio Leme de Oliveira Pinto ${ }^{1}$, Clóvis Yamazato ${ }^{2}$, Fabiano de Mendonça Grandese ${ }^{3}$, Fabio Mastromauro de Oliveira², Felipe de Albuqueroue Araujo Luyten², José Olympio Catão Bastos Júnior ${ }^{2}$, Yoshinobu Nagasse ${ }^{3}$, René Kusabara ${ }^{4}$

\begin{abstract}
RESUMO
Objetivo: Analisar os resultados da cirurgia de corpectomia e artrodese por via anterior nos pacientes com fratura tipo explosão da coluna toracolombar e com osteoporose, que foram submetidos a tratamento conservador prévio por no mínimo sete meses, sem melhora importante do quadro doloroso. Métodos: Foram tratadas seis pacientes idosas com fratura-explosão da coluna toracolombar em um único nível, depois de, no mínimo, sete meses do trauma, pela via anterior, com corpectomia, uso de enxerto autólogo de costela e fixação com parafusos pediculares nos níveis adjacentes. A dor foi avaliada pela EVA no pré-operatório, três semanas, três meses, seis meses e um anos após a cirurgia. Resultado: As pacientes apresentaram melhora significativa da dor pós-operatória, com diminuição substancial do uso de medicação analgésica. Conclusões: Os autores recomendam a via anterior isolada como método de tratamento para fraturas tipo explosão crônicas em pacientes com osteoporose pela baixa taxa de complicações e pelos resultados satisfatórios encontrados.
\end{abstract}

Descritores: Osteoporose; Fratura; Coluna toracolombar; Resultados.

\begin{abstract}
Objective: Analyze the outcomes of anterior instrumentation for corpectomy and arthrodesis on patients with thoracolumbar burst fractures caused by osteoporosis, who underwent conservative treatment for at least seven months, without pain improvement. Methods: Six elderly female patients were treated by anterior instrumentation for burst thoracolumbar fractures at a single level after, at least seven months from trauma, with corpectomy, using rib autograft and fixation with pedicular screws in adjacent levels. Pain was evaluated preoperatively by VAS and three weeks, three months, six months and one year after surgery. Results: The patients present significant post-operative pain improvement, with great reduction in the use of analgesics. Conclusion: The authors suggest single anterior instrumentation as a treatment method for chronic burst fracture on patients with osteoporosis due to the low rate of complications and satisfactory outcomes.
\end{abstract}

Keywords: Osteoporosis; Fracture; Thoracolumbar spine; Outcomes.

RESUMEN

Objectivo: Analizar los resultados de la cirugía de corpectomía y artrodesis por la vía anterior, en pacientes con fracturas tipo explosión de la columna toracolumbar y, con osteoporosis, que habían sido sometidos a tratamiento conservador previo por lo menos durante siete meses, sin mejora importante del cuadro de dolor. Métodos: fueron tratadas seis pacientes ancianas del sexo femenino con la fractura-explosión de la columna toracolumbar en solamente un nivel, después de, como mínimo, siete meses del traumatismo, a través de la vía anterior, con corpectomía, utilización del injerto autólogo de costilla y fijación con tornillos pediculares en los niveles adyacentes. El dolor fue evaluado con EVA en pre-operatorio, tres semanas, tres meses, seis meses y un año después de la cirugía. Resultado: Las pacientes presentaron mejoría significativa del dolor postoperatorio, con la reducción substancial del uso de la medicación analgésica. Conclusión: Los autores recomiendan la vía anterior aislada, como método de tratamiento para las fracturas tipo explosión crónicas en pacientes con osteoporosis, por la baja tasa de complicaciones y por los resultados satisfactorios encontrados

Descriptores: Osteoporosis; Fracturas; Columna vertebral toracolumbar; Resultados.

\section{INTRODUÇÃO}

As fraturas toracolombares são as fraturas mais frequentes na população em geral ${ }^{1}$. Ocorrem com maior frequência no osso osteoporótico, sejam elas por insuficiência do trabeculado ósseo, geradoras do dorso curvo senil, sejam elas por trauma, causando as fraturas que variam com a intensidade de sua energia. Com o aumento da expectativa de vida da população, as fraturas toracolombares associadas à osteoporose tornam-se mais frequentes ${ }^{2-4}$.

O tratamento das fraturas toracolombares do tipo por explosão ainda é muito debatido entre cirurgiões de coluna e existem muitos estudos que comparam o tratamento conservador com o cirúrgi$\mathrm{co}^{2,5}$. Muitos autores defendem a utilização do método conservador de tratamento, principalmente quando não há déficit neurológicos ${ }^{6}$.

1. Residente do Grupo de Coluna dos Hospitais Municipal do Tatuapé (Cármino Caricchio), Ifor e Igesp. 2. Assistente do Grupo de Coluna dos Hospitais Municipal do Tatuapé (Cármino Caricchio), Ifor e Igesp. 3. Médico Voluntário do Grupo de Coluna dos Hospitais Municipal doTatuapé (Cármino Caricchio), Ifor e Igesp. 4. Chefe do Grupo de Coluna dos Hospitais Municipal do Tatuapé (Cármino Caricchio), Ifor e Igesp. 
Apesar da literatura indicar bons resultados por meio do tratamento conservador, existe grande controvérsia sobre o tratamento das fraturas do tipo explosão na coluna toracolombar ${ }^{7}$.

Visando a melhora da qualidade de vida desses pacientes, os autores sugerem a via anterior como acesso único para tais fraturas, com uso de síntese mínima, descompressão efetiva do canal medular e consequente melhora dos sintomas. Portanto, o objetivo deste estudo é analisar os resultados deste procedimento em relação à dor dos pacientes portadores dessa modalidade de fratura.

\section{MÉTODOS}

Foram submetidas ao tratamento cirúrgico seis pacientes do sexo feminino, idosas com fratura do tipo explosão da coluna toracolombar, que foram submetidos ao tratamento conservador por no mínimo sete meses e que apresentavam dor de grande intensidade, necessitando do uso de altas doses de opioides.

Todas as fraturas foram consideradas como sendo do tipo A3 da classificação de Magerl et al. ${ }^{8}$ e, por apresentarem fragmento no canal e por já serem consideradas estáveis pelo tempo passado da lesão, foi optado pela via anterior pura, uma vez que se considerou a compressão como principal causadora do processo álgico ${ }^{8}$.

A mensuração da dor foi realizada com a escala visual analógica (EVA) para quantificar a dor antes e depois da cirurgia. As participantes foram avaliadas no pré-operatório, três semanas após a cirurgia e reavaliadas com três meses, seis meses e um ano de seguimento pós-operatório.

O procedimento foi realizado sob anestesia geral, em decúbito lateral direito, sem intubação seletiva, por meio de toracotomia. Foi realizada corpectomia da vértebra acometida, com colocação de 4 ou 5 fragmentos de costela ressecada no local do corpo retirado para apoio anterior. Como não havia intenção de distração e sim de descompressão e por se tratar de fratura antiga e provavelmente estável, optou-se por utilizar síntese com um parafuso em cada corpo vertebral adjacente à fratura acoplados a haste neste segmento. Todas as cirurgias foram realizadas pelo mesmo cirurgião.

O material de fixação utilizado foi o Omega 21 (Biomet) em duas pacientes e Twister (Proind) em quatro pacientes, não tendo sido observado diferenças técnicas para a fixação.

As participantes foram submetidas à fisioterapia respiratória ainda na unidade de terapia intensiva (UTI), com medicação analgésica e antibióticos profiláticos por 48 horas. Eram encorajadas a deambular no segundo dia de pós-operatório, com retirada do dreno no terceiro dia.

\section{RESULTADOS}

A idade das pacientes variou de 57 a 81 anos (média de 70 anos). O tempo de fratura variou de 7 a 18 meses (média de 10 meses). Quatro pacientes apresentaram fratura de T12, uma paciente apresentou fratura em T10 e uma em L1.

No período pré-operatório as pacientes utilizavam medicação analgésica de forma crônica e contínua, com EVA pré-operatório de 9,7. Após o procedimento cirúrgico, observou-se marcante diminuição dos valores médios da EVA. Com três semanas de pós-operatório o valor médio da EVA foi de 6,1. Além disso, observou-se queda gradativa dos valores médios apresentados pela EVA, que em um ano de pós-operatório atingiu valor médio de 3,0.

O tempo médio de cirurgia foi de 2 horas e 40 minutos, com volume médio de sangramento de $410 \mathrm{ml}$ e não houve necessidade de transfusão sanguínea tanto no intra como no pós-operatório.

O período de internação foi de 6 dias em média, com permanência de um dia em UTI para melhores cuidados de enfermagem.

Todas as pacientes apresentavam processo crônico de osteoporose, sendo que uma delas apresentava hepatite $\mathrm{C}$, o que a impedia de receber tratamento medicamentoso para a disfunção óssea. Todas as outras faziam uso de alendronato semanal e/ou cálcio suplementar.

As complicações pós-operatórias apresentadas foram leves: uma paciente permaneceu com queixa de dispneia leve por quatro semanas, enquanto outra apresentou deiscência do terço posterior da incisão, com infecção superficial, tratada com antibióticos por duas semanas, até cicatrização da ferida.

Em relação ao exame neurológico, uma paciente foi classificada como Frankel D no pré-operatório, e permaneceu sem alterações mesmo após o tratamento cirúrgico. O restante das pacientes foram classificadas como Frankel E no pré-operatório e mantiveram essa condição após o procedimento, indicando a ausência de deterioração neurológica.

\section{DISCUSSÃO}

As fraturas agudas da coluna toracolombar nos indivíduos jovens apresenta classificação e tratamento melhor definido desde a publicação do artigo de Denis ${ }^{9}$ aprimorada pela classificação preconizada por Magerl ${ }^{8}$, utilizando-se para tanto as vias anterior, posterior ou combinada para tal tratamento.

$\mathrm{Na}$ literatura não se encontram muitos trabalhos sobre o tratamento cirúrgico das fraturas crônicas em pacientes idosos, de modo que se observa uma lacuna no que diz respeito ao tratamento cirúrgico destes pacientes que apresentam frequentemente fraturas da coluna, únicas ou múltiplas, por apresentarem osteopenia ou osteoporose. Tais fraturas são muitas vezes de difícil diagnóstico, pela dificuldade de avaliar quando ocorreu ou qual o trauma levou à fratura, uma vez que tais pacientes apresentam histórico de quedas diversas.

Wood et al. ${ }^{10} \mathrm{e}$ Tropiano et al. ${ }^{11}$ mostram bons resultados com o tratamento conservador em pacientes com fraturas toracolombar e afirmam que não há vantagens do tratamento cirúrgico sobre o tratamento incruento, e que o tratamento conservador seria o mais seguro. Apesar disso, Wood et al. $^{10}$ relataram que $8 \%$ dos pacientes apresentavam dor $8 \%$ e Tropiano et al. ${ }^{11}$ relataram $12 \%$ dos pacientes com dor intratável.

Assim como constatou Avanzi, ${ }^{12}$ os pacientes com fratura da coluna vertebral muitas vezes são operados tardiamente, fato que ocorre ainda mais nos idosos, principalmente pela falha no diagnóstico precoce, problemas clínicos associados ou ainda falta de recursos para o tratamento cirúrgico. Muitos destes pacientes não apresentam queixa bem definida, dificultando o diagnóstico, sendo mal orientados nos locais de pronto atendimento, na maioria das vezes com diagnóstico realizado tardiamente, dificultando a indicação terapêutica a ser seguida.

Todas as pacientes do estudo apresentaram diagnóstico tardio, tendo recebido tratamento medicamentoso, fisioterápico e/ou acupuntura, sendo posteriormente encaminhadas ao cirurgião de coluna. Observamos em nossa casuística que todas as pacientes evoluíram com uso contínuo de analgésicos, onde cinco faziam uso de opioides.

As pacientes foram submetidas a tratamento cirúrgico por via anterior isolada. Isso porque estudos biomecânicos mostram que a maior parte das forças de compressão axial são absorvidas na região anterior da coluna, ${ }^{13}$ além de existir a dificuldade de fixação dos implantes nas vértebras osteoporóticas pela via posterior ${ }^{2,3}$. Não foi observada falha ou soltura dos implantes e por isso concluímos que os fragmentos de enxerto de costela foram suficientes para a obtenção do apoio anterior ou a fratura já se encontrava estabilizada do ponto de vista mecânico, com a descompressão do canal tendo sido a responsável pela melhora clínica das pacientes. A literatura mostra que não há grande diferença em relação aos resultado de longo prazo entre pacientes tratados pela via anterior ou posterior isoladamente ${ }^{14,15}$.

Os resultados de nossa casuística, com pequeno sangramento 
intra-operatório e uso de instrumentação mono-segmentar, obtendo-se consolidação óssea, descompressão satisfatória e baixa taxa de complicação nos permite concluir que a técnica utilizada de forma isolada é eficiente para o tratamento dessa modalidade de fratura nos pacientes idosos. A abordagem anterior permite ampla área de trabalho sobre a vértebra, que é obtida após o colabamento do pulmão ${ }^{16,17} \mathrm{~A}$ ausência de soltura ou falência do material de síntese empregado após um ano, com um parafuso em cada corpo vertebral adjacente à vértebra fraturada indica que a fixação, juntamente com os fragmentos de costela utilizados como enxerto ósseo foram suficientes para suportar as cargas axiais até obtenção da consolidação ${ }^{14}$ evitando-se o uso de espaçadores, que aumentam o custo do tratamento e dificultam a visualização radiográfica no pós-operatório.

\section{CONCLUSÃO}

Os resultados de nossa casuística indicam que a abordagem anterior isolada para o tratamento das fraturas crônicas da coluna toracolombar nos pacientes idosos proporciona bons resultados e pode ser uma boa alternativa de tratamento.

\section{AGRADECIMENTOS}

Agradecemos ao Dr. Valter Penna, pela oportunidade de trabaIhar no Hospital A.C.Camargo, onde conseguimos nos aperfeiçoar na técnica da via anterior em pacientes com tumores e metástases vertebrais e ao Dr. Élcio Landim, que sempre nos encorajou a realizar tal técnica sem um cirurgião de acesso.

\section{REFERÊNCIAS}

1. Steib JP, Aoui M, Mitulescu A, Bogorin I, Chiffolot X, Cognet JM et al.. Thoracolumbar fractures surgically treated by "in situ countouring." Eur Spine J. 2006; 15:1823-32.

2. Uchida K, Kobayashi S, Matsuzaki M, Nakajima H, Shimada S, Yayama T, et al. Anterior versus posterior surgery for osteoporotic vertebral collapse with neurological déficit in the thoracolumbar spine.Eur Spine J. 2006; 15:1759-67.

3. Arciero RA, Leung KYK, Pierce JH. Spontaneous unstable burst fracture of the thoracolumbar spine in osteoporosis. Spine.1995; 14:114-7

4. Lee YL, Yip KM. The osteoporosis spine. Clin Orthop. 1996; 323:91-7.

5. Frost HM. Clinical management of the symptomatic osteoporotic patient. Orthop Clin N Am. 1981; 12:671-81.

6. Li-Yang Dai, Lei-Sheng Jiang, Sheng-Dan Jiang. Conservative treatment of thoracolumbar burst fractures: A long-term follow-up results with special reference to the Load Sharing classification. 2008; 33,(23): 2536-44.

7. Yang H, Shi JH, Ebraheim M, Liu X, Konrad J, Husain let al. Outcome of thoracolumbar burst fractures treated with indirect reduction and fixation without fusion. Eur Spine J. 2011; 20(3): 380-6.

8. Margel F, Aebi M, Gertzbein SD, Harms J, Nazarian S. A comprehensive classification of thoracic and lumbar injuries. Eur Spine J. 1994; 3:184-201

9. Denis F. Spinal instability as defined by the three-column spine concept in acute spinal trauma. Clin Orhtop 1984; 189:65-76.
10. Wood K, Buttermann G, Mehbod A, et al. Operative compared with nonoperative treatment of a thoracolumbar burst fracture without neurological deficit: a prospective, randomized study. J Bone Joint Surg Am. 2003:85: 773-81.

11. Tropiano P, Huang RC, Louis CA. Functional and radiograph outcomes of thoracolumbar and lumbar burst fractures managed by closed orthopaedic reduction and casting. Spine. 2003; 28: 2459-65.

12. Avanzi $O$, Chih $L Y$, Meves R. Avaliação do tratamento cirúrgico tardio na fratura toracolombar tipo explosão. Coluna/Columna. 2002;1(2):88-93.

13. Harms J, Stoltze D. The indications and principles of correction of post-traumatic deformities. Eur Spine J. 1992;1(2):142-51.

14. Defino HLA, Scarparo P, Fixação monossegmentar combinada anterior e posterior no tratamento das fraturas da coluna toracolombar. Coluna/Columna. 20076:65(1):3193-4158

15. Cruz CP. Acesso anterior versus acesso posterior no tratamento cirúrgico de fraturas torácicas e lombares. Coluna/Columna. 2006:5(2):71-6.

16. Mcdonough PW, Davis R, Tribus C, ZdeblickTA. The Management of Acute Thoracolumbar Burst Fractures with Anterior Corpectomy and Z-Plate Fixation. Spine. 2004; 29(17):1901-9.

17. Bence T, Schreiber U, Grupp T, Steinhauser E, Mittelmeier W. Two column lesions in the thoracolumbar junction: anterior, posterior or combined approach? A comparative biomechanical in vitro investigation. Eur Spine J. 2007; 16:813-20. 